\title{
Automated Education: Tendency for Scientific Approaches Convergence
}

\author{
Victor Uglev ${ }^{1}$, Dmitry Sukhinin ${ }^{2}$ \\ ${ }^{1}$ Robotics and Artificial Intelligent Lab, Siberian Federal University, Zheleznogorsk, Russia \\ 2 Institute for Computational Neuroscience, University Medical Center Hamburg-Eppendorf, Hamburg, Germany \\ uglev-v@yandex.ru,d.sukhinin@gmail.com
}

\begin{abstract}
In this paper we discussing the possibility of the complex use of advances in area of pedagogic, psychology, neuroscience and informatics for the creation of teaching and learned systems of new generation. It is shown that the theoretical basis of automated education people (educational systems and programmathematical models) and machines (neural network simulation and intellectual robotic systems) can be integrated into a new scientific
\end{abstract} direction.

Index Terms - education, cognitive psychology, artificial intelligence, neuroscience, computational neuroscience, neuroinformatics, cognitive maps

\section{Introduction}

Education as the process of acquiring of new knowledge has been widely elaborated in many scientific disciplines: pedagogic, psychology, neuroscience and informatics.

Today processes of automation help not only to conduct education with use of traditional and self organized approaches (in this case they are tools) but they help to create learning systems (in this case these systems are being the target of education process). Until recently, technologies and approaches to educate people and intellectual agents (limited to robots) were based on different methodological bases. But successes in aforementioned scientific disciplines allow to mark the tendency for their convergence around the main driving force - informatics. Let us discuss this in more detail.

Education of a human with use of e-learning courses, automated educational systems, program-mathematical models and virtual reality allowed to come really close to the solution for the problem of forming the image of a student inside computer. The model of a student, together with the model of a teacher, a tutor, the teaching situation and the logic of their interaction already overcome the boundaries of classical knowledge engineering and require a much elaborated methodological basis. In a new way possibilities of automated reeducation are being perceived with a great novelty as aimed to bring together logic of human expert decision-making and a computer.

Let us continue with the analysis of advances in the key scientific disciplines discussed above and show the main role of informatics as the fundamental and the gravitation force in development of teaching and learned systems of new generation.

\section{Components of Learning}

A. Pedagogical Component

Since the time of first attempts to automatize educational process almost one hundred years have passed (the programmed learning approach by B. Skinner and N. Crowder). During this time focus has changed from simple algorithms for learning trajectory control to the attempts to implement all basic didactic principles. Most progressive tools in this field have became Intellectual (in terms of Artificial Intelligence) Automated Educational/Training Systems (AES), that includes both a theoretical modules (electronically lectures, thesaurus, reference books), and practical modules (electronic workbooks, the program-mathematical models, virtual laboratory and etc). But, regardless of how widely this e-learning platforms (for example Moodle) are used, they are still fundamentally inferior to humans in terms of teaching and tutoring capabilities.

In recent years there has been increased interest of researchers to ideas of individualization, tutor's work automation and dialogue organization with the AES users. All of this leads to the rethinking of methodological bases of computer-based learning: students begin to be perceived not as a uniquely "programmed" object, but as individuals, having their goals, interests and the logic of reasoning. In a certain sense, model of each student - is no longer a white box (in terms of system theory), but rather a gray box, that has its own aims and behavior model. Consequently, the AES will be constantly updating student image, exploring it and adapting to its psychological characteristics, by the analogy with actions of a human teacher. Student model and teacher/tutor model in the learning system will gravitate towards self-sufficiency, claiming the role of intelligent agents, that can interact in a virtual environment.

\section{B. Psychological Component}

During the performance of automated education by intellectual systems it is necessary to apply vast knowledge from cognitive psychology [1] and take to account specifics of human-computer interactions [2].

In order to make automated training successful, it should provide a zone of proximal development (by L. Vygotsky [3]), simulating a job of a human teacher and a subject tutor. It entails the need to modernize the methodological basis of automated learning, adapting classical didactics J. Komensky to the specific interaction between man and machine. Research 
in the field of logic reasoning P. Anokhin (afferent synthesis process [4]) and attempts to simulate they in intellectual AES [5] show that the decision-making mechanism for the management of the educational trajectory can successfully automate on the level of operating categories and modes, that formed a particular learning situation.

For the last 25 years the emergence of compute methods of cognitive visualization (graphics) have allowed to appear the enhanced perception capability of images by student and learning machines [6, 7]. At the same time problems of intensification are being solved for presenting didactic material as well as for navigation and reasoning solutions (including the making cognitive maps, see Fig. 1).

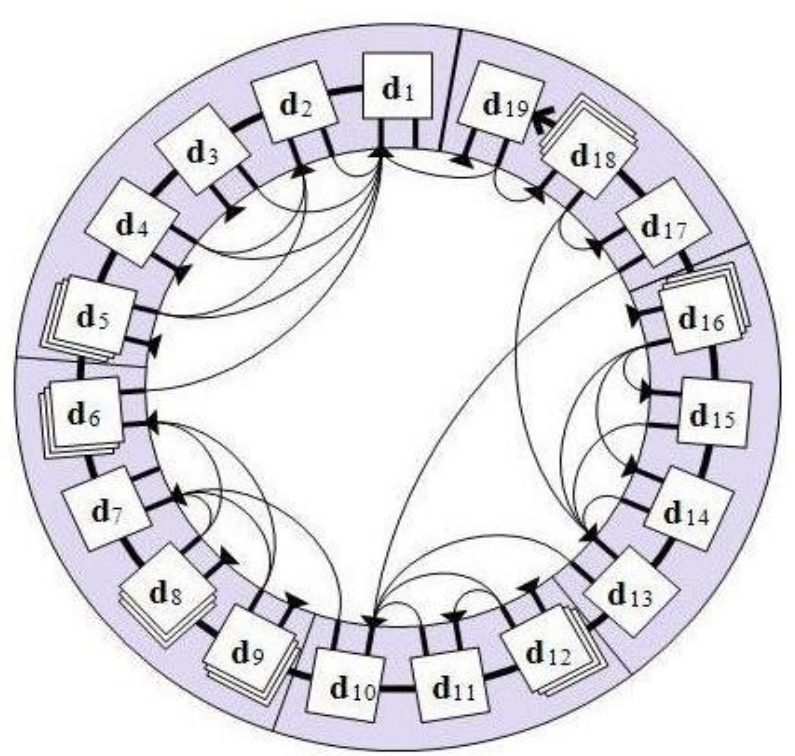

Fig. 1 Cognitive Maps of Knowledge Diagnosis example for some e-course in AES [8].

Another notable trend in cognitive psychology has become the interest to use gamefication approaches for learning in Internet [9]. With the development of virtual communication facilities (including telepresence and 3D-worlds) it is became possibly to develop methods for action-driven learning, that allow procedural knowledge to be strengthened by engaging the learner in the process of learning tasks at all levels of perception.

\section{Neuropsychological Component}

Despite to all accumulated experience by humanity in teaching and education, and even taking to account recent success in area of neurocognitive sciences - the understanding of education process in humans at the level of neuronal networks biology still stays poorly understood and requires a mass of efforts and multidisciplinary approach. However, even today we know that at the basic level the process of learning in the human brain and in different animal species is just a creation of new synaptic connections among neurons with use of intracellular intricate molecular signalization, DNA replication and external exchange of neurotransmitters and neuromediators [10].

On the other hand, the process of learning at level of neural networks is an active process with complicate transmission of signals in horizontal and vertical direction of hierarchy [11]. Attempts to describe this process, starting with compilation of cognitive maps [12] and up to large scale simulations (for example: Blue brain project, http://bluebrain.epfl.ch/), allowed to obtain masses of important data, but still, did not make it absolutely clear what is really going on with topology of neuronal networks and its role in learning.

Main problems in studying brain are due to its complexity and ethical limitation on direct (invasive) experiments with humans and animals.

As a solution to these problems, researchers started to study brains with computer models. Hence computational neuroscience has appeared. For its short story (notably tightly connected to the development in computer science, machine learning via imitation of artificial neuronal networks), computation neuroscience produced significant results that show that even simple models can successfully explain or shed light on complex processes taking place inside brains [13].

Modeling take place on different scales from molecular to the scale of cognitive functions. The most promising scale for the entire discipline of neuroscience is the level of micro and macro structural connectivity of the brain. In this direction the huge work has been done already. For the several relatively simple species like C.elegance and Drosofila the full connectome at the neuronal level was obtained. At the level of brain regions so called macroconnectome was obtained for the cat, macaque monkey (see Fig. 2) and in additional macroconnectome for the ferret (Mustela putorius) will be published soon [14]. Both levels of detalization, micro and macro, can be used further for large-scale brain simulations via interconnections among different neuronal models and connectivity among them.

Today, one of the most sophisticated models of the brain is SPAWN simulation from Canadian group [15]. This simulation includes several models and shows many of the brain capabilities such as image recognition, working memory and even forgetting and hesitation. One of many important features of this model is that is conducted inside virtual reality, where with use of a screen, some information is being conducted into an artificial eye. After processing this information, the result can be outputted as a written text with use of an artificial hand which has several degree of freedom. Usage of virtual reality gives many opportunities to develop and to debug models neuronal nets, cognitive systems and robots that are based on biologically realistic principles of the brain inner mechanics. Controllable environment of virtual reality with limited and known number or parameters fosters to conduct experiments by lowering their costs, preparation time and helps in consequent analysis. 


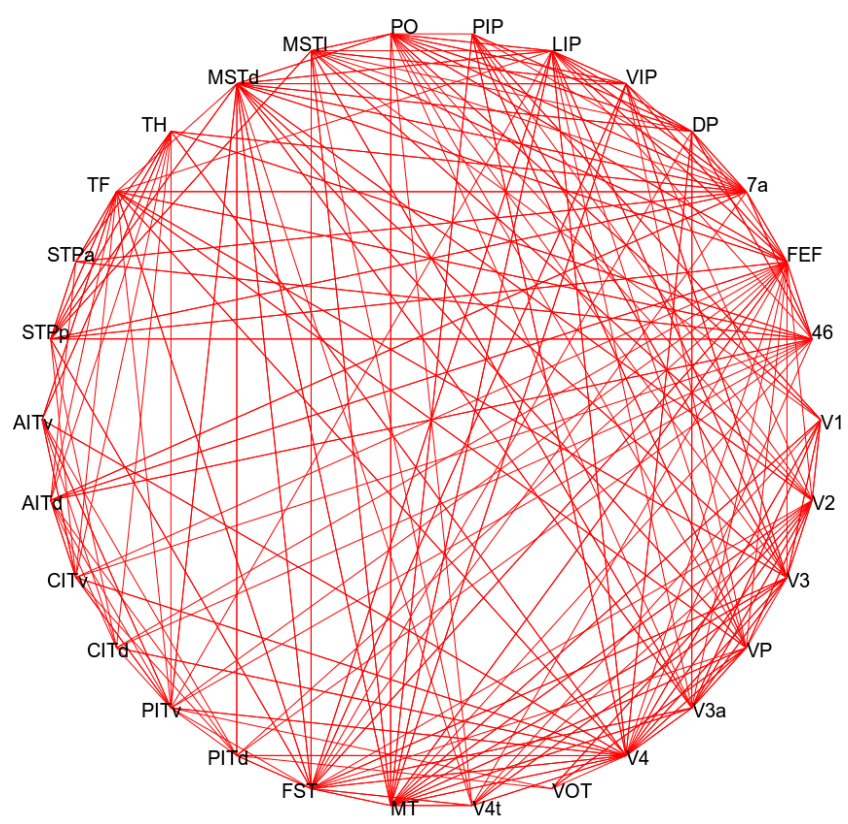

Fig. 2 Structural connectivity of macaque monkey visual cortex (reconstruction of data from [16]).

To conclude, in order to understand of the learning and hence educational process, it is necessary to study first the basic substrate, the brain that can actually learn new thing by altering its own structure at synaptic level as well as at the level neuronal networks. Modeling on a computer such processes - brains connectivity is an essential and necessary way (and even probably the only one due to brains complexity) to shed light on complex organization of structure of the brain and relation of this structure to brain functions. In turn, the virtual environment is perfect tool for testing of all features of such models.

\section{Technical and Technological Solutions}

Ever increasing performance capabilities of computers and miniaturization of their components allows today to create such automated systems that can bring absolutely new types of teaching and learned systems.

Successes in creation of AES at first hand related to advances in artificial intelligence, where the technology of intellectual agents allows to direct pedagogical influence on a student's according to their personality. By combining mechanism of experts systems and wighted ontologies intellectual AES it is became possible to organize individual learning space. In such system interaction between a student and the educational system can be organized via advanced natural language interfaces (for example see AMI project [17]). Obviously that inside this approach, the main role will be playing a knowledge base. This knowledge base will contain methodological and structural information about the object of education. In this case AES can be created as virtual educational environment and based on programmathematical models as well as software-hardware robotic systems, which can be oriented to facilitate acquiring of more practical skills (for example see [17]).

Development of learning models of the brain at the first place is based on ideas brought from neuroinformatics [18] which is one of subareas of AI. Simulation can be run on specialized equipment (for example an analog scheme Anadigm AN221E04 can be used), as well as with use of standard computers which, in turn can significantly simplify development and deployment of system for simulation of neuronal networks (for reference sake, modified version of Neuron software which can be run on all main operation systems is used by the Blue Brain project, http://www.neuron.yale.edu/). However the use of standard computers for this task has fundamental limitation: to perform large scale simulation it is necessary to use supercomputers which consume huge amount of power with respect to one step of simulation. To solve this issue it would be plausible to develop and to use specialized processors with only one function dedicated to emulate work of neurons [19].

In the area of creating virtual environments for scientific and another non-commercial goal there are many powerful and functional systems for 3D modeling (for example Blender, http://www.blender.org/) and for game development (for example Unity3d, https://unity3d.com/). There are as well specialized systems for testing robots hardware inside virtual environment which is closely imitates the real world (see Gazebo, http://gazebosim.org/). But this approach is oriented on simulation of educational principles rather than to use semantics of didactic material.

\section{Convergence of Scientific Approaches}

From the first sight, technical and technological solutions for teaching and learned systems have significant differences but, obviously it is clear that software and hardware components of information systems have mutual influence at each other.

Even more, many researchers in area of artificial intelligence do not draw principle line between virtual agents and their hardware variant (for example robots), from position of describing behavioral and decision making model [20].

It is very important that recently number of multidisciplinary projects has risen for studying processes of education in area of humanitarian sciences (cognitive psychology and pedagogic), biomedical sciences (neuropsychology and computational neuroscience) and in area of technical sciences (neuroinformatics and robotics). It is clear that in order to obtain new results it is necessary to perform projects in overlaps of these scientific disciplines. It is possible that in near future a new scientific discipline will appear, formed as the convergence of previously mentioned scientific disciplines or it will migrate to the area of study of cognitive informatics [21] without losing important humanitarian part. Time will show if different researchers groups from different scientific disciplines will be able to organize such complex projects. In any case it is became increasingly important to study mechanisms of education 
process oriented to automatize this process. Let us list possible direction of works:

- detailization and perfection of artificial brain model, its learning capabilities for solving practical tasks,

- search in huge amounts of data and information and consequent extraction of knowledge from short-term or longterm memory of teaching or learned system,

- recognition in real time direct and secondary communication signals received in natural language form and producing a response in form of a dialog,

- locomotion that provides efficient solution for tasks of cinematic at the level of reflexes during movement control of robotic system,

- education with use of AES and robots and vice versa.

Finally, understanding of educational process and its use in applied tasks of automation will provide significant results for humans educational process aimed at increase of educational level and individual development of a person as well as for better development of entire humanity.

\section{Acknowledgment}

This research is supported by the Ministry of Education and Science of the Russian Federation in Siberian Federal University (contract № 02.G25.31.0041).

\section{References}

[1] R. Solso, ed., Mind and Brain Science in the 21st Century, The MIT press, 1999.

[2] E. Mashbits, Psychology-pedagogical problems of computerizing learning, Moscow, Pedagogica, 1988. Available at: http://pedlib.ru/ Books/6/0442/ 6_0442-1.shtml (in Russian).

[3] L. Vygotsky, (1935) "Dynamics of cognitive development in relation to student learning," Mental development of children in the learning process, Moscow, 1935, pp. 33-52 (in Russian).

[4] P. Anokhin, Biology and Neurophysiology of the Conditioned Reflex and Its Role in Adaptive Behavior, Pergamon press, 1974. Available at: http://www.evolocus.com/Textbooks/Anokhin1974.pdf.

[5] V. Uglev, "Implementation of Decision-making Methods in Intelligent Automated Educational System Focused on Complete Individualization in Learning," AASRI Procedia, vol. 6, 2014, pp. 66-72. DOI 10.1016/ j.aasri.2014.05.010.

[6] Yu. Nechaev, A. Degtyarev, A. Boukhanovsky, "Cognitive computer graphics for information interpretation in real time intelligence systems," LNCS, vol. 2329, 2002, pp. 683-692. DOI: 10.1007/3-54046043-8_69.
[7] V. Uglev, T. Kovaleva, "An application of cognitive visual representation as a tool to support individual education," Science and Education, no. 3, 2014. Advailible at: http://technomag.bmstu.ru/ en/doc/700661.html. (in Russian)

[8] V. Uglev, "The Cognitive Maps of Knowledge Diagnosis," Open and Distance Learning, no. 48, 2012, pp. 17-23. Available at: ido.tsu.ru/other_res/pdf/48-4.pdf. (in Russian)

[9] C. Muntean, "Raising Engagement in E-Learning through Gamification," The 6th International Conference on Virtual Learning ICVL, 2011, pp. 323-329. Available at: http://icvl.eu/2011/disc/icvl/documente/pdf/met/ ICVL_ModelsAndMethodologies_paper42.pdf.

[10] E. Kandel, "The molecular biology of memory storage: a dialog between genes and synapses," Bioscience Reports, vol.. 21, no. 5, pp. 565-611, 2001. Available at: http://www.signallake.com/innovation/ Kandel120800.pdf.

[11] J. Fuster, Cortex and mind: Unifying cognition, Oxford university press, 2003.

[12] E. Tolman, "Cognitive maps in rats and men," Psychological Review, vol. 55, pp. 189-208, 1948. DOI: 10.1037/h0061626.

[13] R. OReilly, "Biologically Based Computational Models of High-Level Cognition," Science, vol. 314, no. 5796, pp. 91-94, 2006. DOI: $10.1126 /$ science. 1127242 .

[14] D. Sukhinin, C. Hilgetag, "Building the Ferretome. Front. Neuroinform.," Conference Abstract: Neuroinformatics, 2013, in press. doi: 10.3389/conf.fninf.2013.09.00007.

[15] C. Eliasmith, "A large-scale model of the functioning brain," Science, vol 338, no 6111, pp. $1202-1205$, 2012. DOI: 10.1126/ science. 1225266 .

[16] D. Felleman, D. Van Essen. "Distributed hierarchical processing in the primate cerebral cortex," Cerebral cortex 1(1), pp. 1-47, 1991. Available at: http://www.cns.nyu.edu/ tony/vns/readings/fellemanvanessen-1991.pdf.

[17] V. Uglev, A. Naslimov, K. Kondratyev. "Adaptive control of registration area by the acoustic signal in AMI systems," International Siberian Conference on Control and Communications (SIBCON), 2013, pp. 1-4. DOI: 10.1109/SIBCON.2013.6693651.

[18] A. Khnykin, N. Laletin, V. Uglev, "Applying Zheleznogorsk Robotics for Learning Children with Disabilities," LNCS, vol. 6693, 2011, pp. 167-171. DOI: 10.1007/978-3-642-21303-8_23.

[19] G. Shepherd, "The Human Brain Project: neuroinformatics tools for integrating, searching and modeling multidisciplinary neuroscience data," Trends Neurosci, vol. 21, 1998, pp. 460-468. DOI: 10.1016/S0166-2236(98)01300-9.

[20] P. Merolla, et al, "A digital neurosynaptic core using embedded crossbar memory with $45 \mathrm{pJ}$ per spike in $45 \mathrm{~nm}$," Custom Integrated Circuits Conference (CICC) IEEE, 2011, pp. 1-4. IEEE, 2011. DOI: 0.1109/CICC.2011.6055294.

[21] S. Russel, P. Norvig, Artificial Intelligence. A Modern Approach (2nd ed.), Prentice Hall, 2003.

[22] Y. Wang et al, "A Doctrine of Cognitive Informatics (CI)," Fundamenta Informatic, vol. 90, no. 3, 2009, pp. 203-228. DOI: 10.3233/fi-20090001 . 